\title{
DIPHTHERIA AT A HOSPITAL CENTER
}

\author{
Moses Barron and George H. Bigelow
}

From the Base Laboratory, Hospital Center, Allerey, Saone et Loire, France

Havard" states that diphtheria is "a disease of minor importance in the Army." He quotes statistics to support this view. Keefer ${ }^{2}$ and others, however, find that an epidemic of this disease is at times very difficult to control. It has been the experience of the writers at the Hospital Center, Allerey, Saone et Loire, France, that it requires at times considerable effort, ingenuity and cooperation between the laboratory, ward surgeons, nursing corps and patients, to bring about the desired results. In one-story wooden barracks and tents, covering less than one square mile of space, there were over 17,000 patients and 3,000 personnel at the time that the disease was at its maximum. It is evident that the crowding was very great, and this condition always hampers the control of a contagious disease.

\section{EPIDEMIOLOGY}

The first case of diphtheria on record at this Center occurred in Ward V, Base Hospital 26, Sept. 24, 1918. There were two more on Oct. 1, one on the 5 th, one on the 8 th, three on the 9 th, and so on until in the period of Nov. 10 to Dec. 8, from one to seven cases were reported daily. The incidence of clinical cases and carriers at each base hospital is shown in chart 1 . Chart 2 is a composite of all the cases in the entire Center. The three hospitals (26, 25 and 49) in which the first cases appeared, were the ones in which more than two-thirds of the entire number of cases developed. It should be borne in mind that since these three hospitals were the first to arrive at this Center, they received the largest number of patients. In these hospitals several nurses and ward men who had been known to be carriers in the States were again identified as such here, but only after the infection had been under way for some time and the culturing had become general. Such chronic carriers might have been factors in introducing the disease at this Center, but it is also possible that they were reinfected here. It may perhaps be unjust that a person once found to be a diphtheria carrier should thereafter always be blamed for any diphtheria developing in the vicinity, but the possibility of course cannot be denied. One of the supposed carriers had only a true Hoffman's bacillus morphologically and culturally, since it did not ferment any of the sugars.

Very early in the epidemic carriers were found with striking frequency among the gassed cases. A number of these cases, diagnosed as diphtheria because of positive throat cultures, came to postmortem examinations and

Received for publication March 13, 1919.

1 Military Hygiene, p. 91.

2 Keefer, F. R., Friedberg, S. A., and Aronson, J. D.: Jour. Am. Med. Assn., 1918, 71. D. 1206. 
showed either no membrane at all or only a small and clinically insignificant one. Yet from the larynx of these cases practically pure cultures of typical B. diphtheriae were obtained. The relative frequency with which such cases were encountered suggested a second possible mode for the introduction of the organism at this Center.

The first ward to be under quarantine for any length of time, due to the constant discovery of new carriers, was the pneumonia ward at base hospital 26 , where there were a large number of gassed cases. The same was true in base hospital 56. In base hospital 25 the first cases of diphtheria appeared in the gassed wards. At one time there were 75 known diphtheria carriers (chart 5, B) of whom $331 \frac{1}{3} \%$ gave histories of gassing; whereas, of the 35 clinical cases only $5.7 \%$ gave such a history. As a possible explanation of the marked preponderance of carriers over clinical cases, it is suggested that the inflammatory changes in the respiratory mucous membrane incident to gassing produces an environment favorable for bacterial growth. The pneumococcus and particularly the streptococcus are invasive organisms frequently causing pneumonia in such cases, but the diphtheria bacillus is relatively noninvasive. Consequently, while the antitoxin producing power of the patient remains unimpaired, diphtheria bacilli may be present in large numbers without causing the disease. Such cases are carriers. It was impossible to go on with this phase of investigation since with the cessation of hostilities no more gassed cases arrived.

Crowding, which means droplet infection, was probably the chief factor in the spread of diphtheria at this Center. During October and early November all wards were greatly overcrowded. Designed for 50 beds, they held 70 . To many of the wards were attached one or more tents of 50 beds each, with only just space enough to crowd between every second bed. In the wards, two patients had to share the same table. There were barely sheets enough to cubicle the acute respiratory wards.

When the pneumonia ward of base hospital 26 was first quarantined it was very striking how, over a period of some 2 weeks, carriers continued to appear in two of the tents connected with the ward, whereas the third tent remained uninfected, though cultured as often as the others. This result can be explained only by strict isolation of the uninfected tent from those which were infected. or, due to the crowding, had the organism once been introduced, it would undoubtedly have spread from one end of the tent to the other in a comparatively short time, just as it had in the other two.

The indirect spread of infection by hands and fomites as opposed to direct spread by droplet infection has been known for a long time. ${ }^{3}$ Fumigation in all its aspects is intended to combat this. But of late the idea that a contagious patient in bed may infect the outmost corners of his room has been replaced by that of direct droplet infection incident to coughing, talking, sneezing, etc., so that only objects in immediate contact with a patient are considered dangerous. Supreme emphasis is therefore placed on the masking of all in attendance. The pendulum has swung a little too far, as our investigation would suggest, from indirect transmission through hands and fomites to direct transmission by means of mouth to mouth droplet infection. This phase of the investigation was suggested by Lieut.-Col. Witherspoon, and it seemed that in the typical diphtheria bacillus we had an unusual opportunity for study, since this organism, we thought, could be recognized in culture by its morphology alone. Also we had here a considerable number of clinical cases and contacts under far better control than is possible in civil life.

${ }^{3}$ Park, W. H., and Williams, A. W.: Pathogenic Micro-organisms, 1917, p. 297. 
Provisionel. Maspital.4.

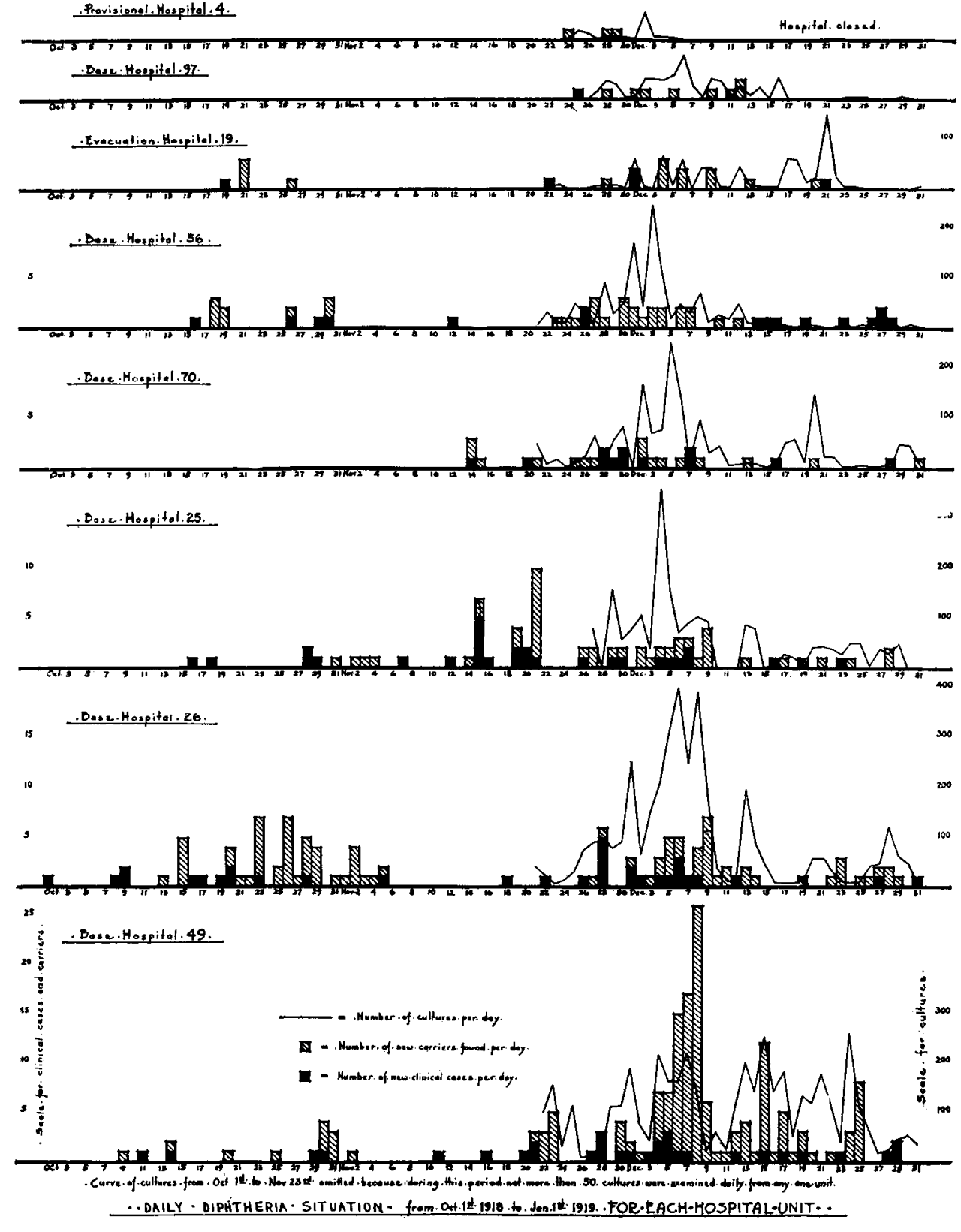

Chart 1.-It should be noted that base hospitals 49,25 and 26 furnished most of the clinical cases and carriers. Up to Nov, 6, three to four carriers were found at base hospitals 49 and 26 for each clinical case, whereas, compared with the number of clinical cases, base hospital 26 showed few carriers. This may in part be accounted for by the less general culturing at this hospital at this time. The clinical case at base hospital 26 recorded on Oct. 1 was the first case at this Center and should have been recorded on Sept. 24, had the chart permitted. The first carrier at provisional hospital 4 was sent to base hospital 56 as a clinical case, but it was decided to be merely a follicular tonsillitis with a positive culture. On Dec. 6, 7 and 8 the marked increase in carriers at base hospital 49 was due to the great prevalence of the atypical forms. The same were found Dec. 8 at base hospital 26 , when the carelessness was shown in taking cultures. These were never reported positive, since the ward was quarantined and all throats cultured. The same procedure was adopted at base hospital 49 , as is shown by the marked drop in the curve of cultures Dec. 9 . The most at base hospital 49 , as is shown by the marked drop in the curve of cultures Dec. 9. The most observation ward, which may account for the absence of new carriers. It should be noted that in all instances the sum of chart 1 will not equal chart 2. This is because some 13 of the first clinical cases could not be traced back to the base hospital where they originated, and some few cases were sent in from the convalescent camp, which is not shown on chart 1 . 
The diphtheria wards, both clinical and carrier, were chosen for this investigation, using wards in which no cases of diphtheria had developed as controls. In all, 522 cultures were made. The following procedure was adopted: a sterile swab moistened in sterile broth was wiped over the palm, particular attention being paid to the creases. It was felt that owing to heat and moisture the creases would be most likely to retain the organisms which might be deposited there. The swab was inoculated on Loeffler's blood serum and incubated from 16-18 hours. Working on the principle suggested by Williams, ${ }^{4}$ some 90 cultures were enriched after 8 hours' incubation by inoculating fresh tubes with a loop passed over the surface of the original one. Both series of tubes were examined from 10-12 hours later. The enriched series did not show a constant increase in the number of positives and the procedure was discontinued. A few cultures were first taken from the fingers also, but this was soon abandoned because no additional information was obtained. It was felt that contamination of the fingers could be reasonably assumed once the palm was proved positive.

All the attendants and nearly all the patients were masked in the clinical and carrier diphtheria wards. A few of the bed patients who were cubicled were not masked Occasionally the patients were asked to cough before the cultures were taken. Patients without masks and often patients with masks would unconsciously place their hands on their mouths when coughing. At times they were explicitly told to cough into their hands. This was done routinely toward the latter part of our investigation.

For purposes of comparison patients were divided into two groups: Those in "infected" wards, and those in "uninfected" wards. We use the term "infected" wards to mean wards in which clinical cases or carriers were isolated. Base hospital 56 furnished the "infected" wards because all clinical and carrier cases from the Center were sent there. The term "uninfected" refers to wards in which no clinical case or carrier had been identified at any time; such wards were selected from hospitals in which there had been the least amount of diphtheria. These groups were again divided into "masked" and "unmasked." Unmasked patients were rather hard to find in the diphtheria wards, with the exception of the cubicled bed patients. In the "uninfected" wards chosen, all were unmasked. Fomites were similarly subdivided into "masked" and "unmasked." Only articles used or handled by masked patients were considered "masked" fomites. Such articles included door-knobs, handles on latrine pail covers, pokers, crutches, and personal possessions such as mess-kits, mirrors, rosaries, magazines, foot-rails of beds, etc. The personal possessions of unmasked bed patients were considered "unmasked" fomites. This distinction is entirely arbitrary, since cigaret papers, spoons, mirrors, combs, etc., are used when the mask is off and the patient who is masked at the time of culture may have had his mask off or hanging from one ear only 5 minutes previously.

The typical diphtheria bacillus when present in these cultures was found only after considerable search. They were usually in clumps of three or more, showing polar-staining and parallel or stellate arrangement. The majority were of the smaller forms corresponding to Wesbrook's " $\mathrm{C}$ " or " $\mathrm{D}$ ". The atypical diphtheria bacillus or diphtheroid was similar in morphology to that found in the throat, in the mouth, in the anterior nares, and in several of the wounds. It resembles the diphtheroid described ${ }^{5}$ as the normal inhabitant of the skin.

- Ibid., p. 306.

- Eberson: Jour. Infect. Dis., 1918, 23, p. 1. 
The number of the organisms showing metachromatic polar granules was very surprising. Some of these were often indistinguishable from the large polar-staining diphtheria, though the frequency of a large metachromatic granule at the center made one at once suspicious. Also the majority did not show the characteristic tapering toward the center nor the usual slight curve. When the grouping of these organisms in the smears was considered, the absence of all palisade arrangement was striking, the bacilli often appearing in chains of two, three or even more. Spore-bearing baçilli were found

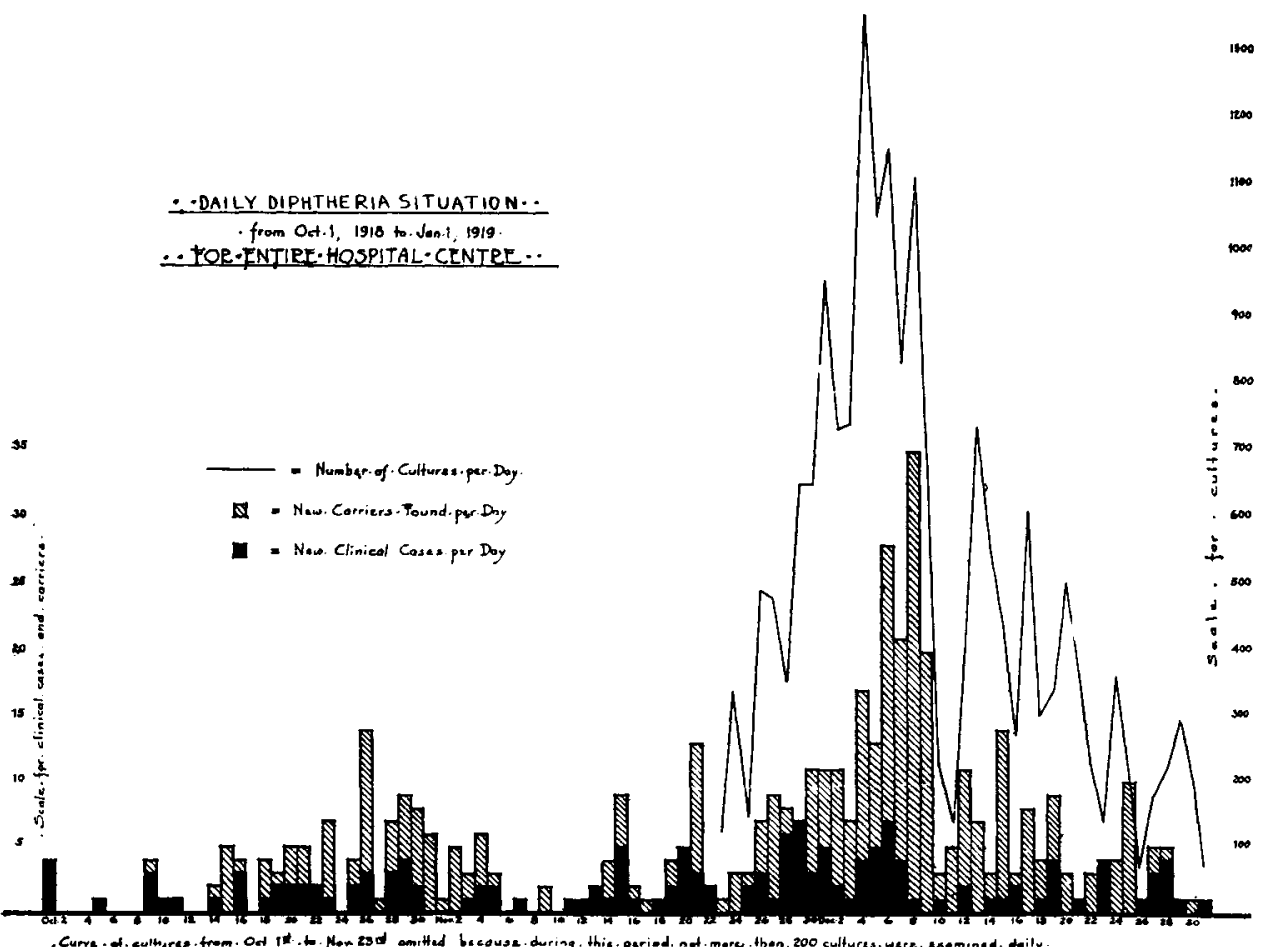

Chart 2.-Note that in general the curve of new carriers found follows that of the number of cultures examined. The marked exception is on Dec. 8, when the atypical form was being found in great numbers at base hospital 49 . In Oct. and early Nov. the culturing was much less general, only those immediately exposed being cultured. Hence, there was at that time a much higher proportion of positives.

in the same smears. These two forms were occasionally the only organisms present. It is, of course, unreasonable to expect to recover an almost pure culture of diphtheria bacilli in cultures from the foot of a bed or from the hand of a patient, even when the patient is known to harbor this organism in his throat. It was felt that the spore-bearing and the polar-staining bacilli were probably only different forms of the same organism-a saprophyte corresponding to Bizzozero's Leptothrix epidermis and not a diphtheroid at all. To prove this, isolation was attempted. Isolation through plating failed because of the extremely spreading character of the growth. Isolation of the spore-bearer through heating, however, was successful. Suspensions of the 
culture in salt solution were heated for half an hour at $70 \mathrm{C}$. and then planted out on Loeffler's blood serum. As controls were used cultures of typical diphtheria from a clinical case which killed a guinea-pig in 36 hours; a culture of typical diphtheria recovered from a larynx at necropsy; a culture from a wound showing type "D 2" organisms. All the controls showed no growth in the subcultures at the end of 72 hours. The subculture of the spore-bearer, however, showed an abundant growth at the end of 24 hours with both the polar-staining and the spore-bearing forms present. These were identical with the original culture. Cultures from the foot of the bed, from a hand, and from a rosary, all showing these two forms, had the same forms in the subcultures after heating. This gave us convincing proof that this polarstaining form was not of the diphtheria group at all, though in occasional instances it would have been extremely difficult to exclude it without the examination following heating.

Chart 3 shows results of the 522 cultures from hands and fomites; $16.1 \%$ of the "unmasked" hands in "infected" wards showed typical diphtheria, while only $6.4 \%$ of the "masked" hands showed it, thus indicating the value of masking in preventing droplet infection of the hands. Of the cultures from unexposed wards, $5.4 \%$ showed a diphtheroid and none typical diphtheria bacilli. The incidence of this diphtheroid in cultures from exposed wards was much higher, $10.3 \%$; in these wards patients were known to harbor a morphologically similar organism. However, since these organisms or similar organisms are normal inhabitants of the skin, the results of the investigation as far as this organism is concerned, may be somewhat misleading, because its presence may be accounted for otherwise than by droplet infection from the throat. But when a typical diphtheria bacillus is found on the hand and at the same time is known to be an inhabitant of the patient's throat, it is reasonable to assume a direct transmission from throat to hand by coughing or sneezing, and thence to fomites by the contaminated hand. As noted above, this study would emphasize the value of masks in preventing droplet infection of hands and probably of fomites, but whether direct droplet infection from mouth to mouth or indirect droplet infection by way of the hands and fomites is the principal mode of spread must still be little more than a matter of individual opinion. However, this does emphasize what has long been knownthe importance of the indirect method of spread of diphtheria. Greater attention must be paid to this manner of disseminating contagious diseases.

A third factor in spread, once the organism had been introduced, was late diagnosis. Because of the considerable number of gassed patients in the hospitals, recognition of laryngeal diphtheria was at times extremely difficult. This was due to the great similarity between the two diseases, not only in the mechanical obstructive symptoms which developed but also in the appearance of the membranous exudates. The severely gassed cases of ten developed fibrino-purulent membranes which covered the lining of the larynx and trachea, extending from the epiglottis down into the bronchi and bronchioles. The aryepiglottic folds and tissues about the ventricles and vocal cords were at times markedly edematous, producing voice changes, hoarseness and mechanical obstruction to breathing. This membranous exudate rarely extended upward into the pharynx and never over the tonsils. But nearly all of the serious cases of diphtheria had severe laryngeal involvement, often without any pharyngeal manifestations, so that even at necropsies it necessitated close scrutiny to differentiate between the two types of lesions. Besides, diphtheria was sometimes superimposed on the laryngitis following gassing. It is there- 

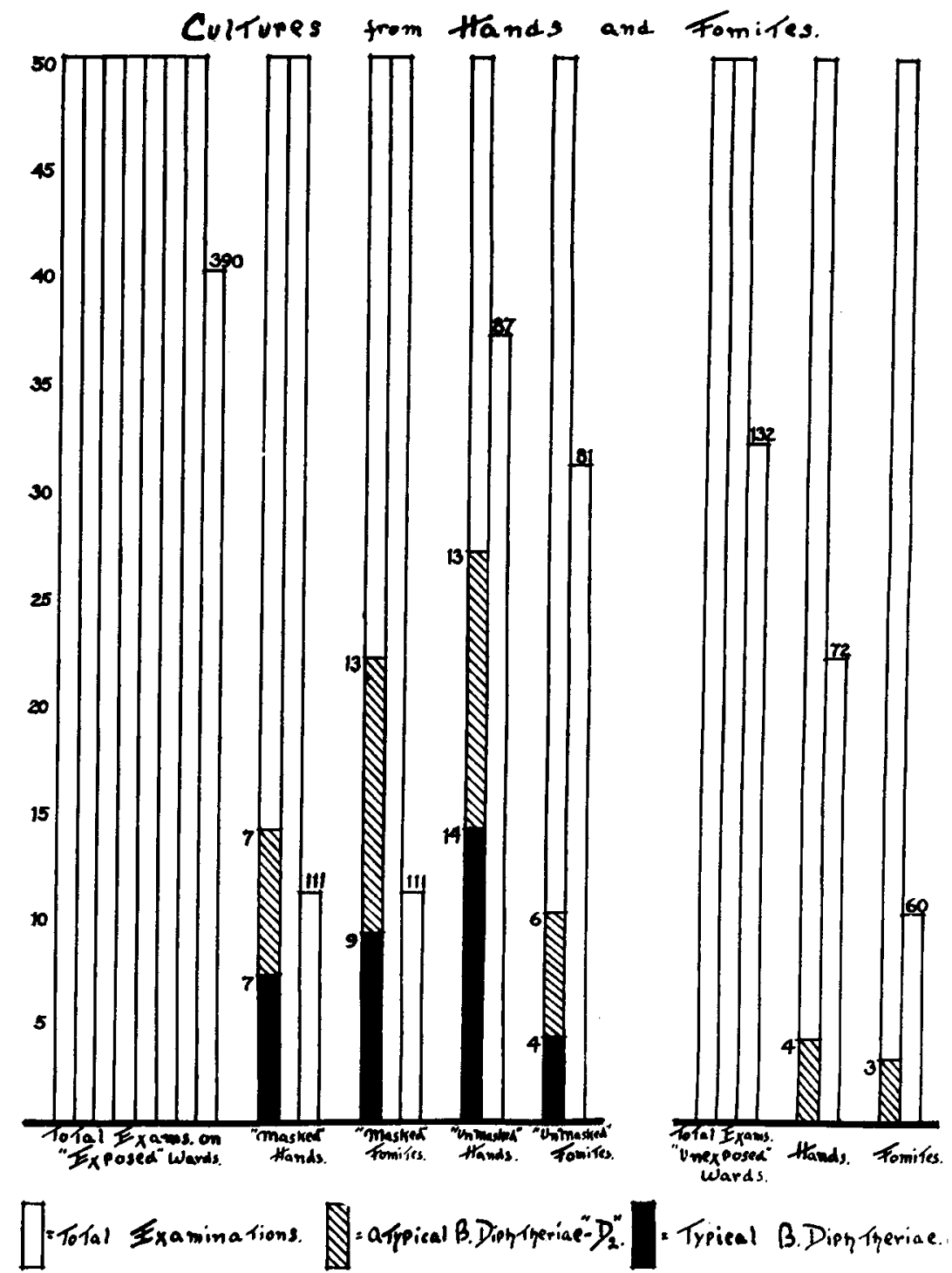

3.- "Exposed" Wards: Typical B. diphtheriae on "masked" hands, 6.3\%; on "masked" fomites, 8.1\%; Typical B. diphtheriae on "unmasked" hands, $16.1 \%$; on "unmasked" fomites, 4.9\%; Atypical B diphtheriae on "masked" hands $6.3 \%$; " "masked" fomites, $11.7 \%$; Atypical B. diphtheriae on "unmasked" hands, $14.9 \%$; on "unmasked" fomites, 7.4\%. "Unexposed" Wards: Atypical B. diphtheriae on "unmasked" hands, 5.9\%; on "unmasked" fomites, 5.0\%. "Exposed" wards were those in which the clinical cases of diphtheria or carriers were treated. "Unexposed" wards were those in which no cases of diphtheria or carriers bad been found at any time. The term "masked" men that the patient whose hands or fomites were cutured wore " versely that he wore no mask. It should be noted that typical B. diphtheriae was found nearly three times as often on the hands of those not wearing masks as on those i earing them. Nearly the same difference was found with the atypical form. With the fomites the "masked" were more often infected. This may be explained in part by the fact that many of the fomites considered "masked" were used only when the patients were unmasked. None of the control cultures on "unexposed" wards showed typical B. diphtheriae. 
fore easily seen that there were great difficulties in differential diagnosis. As a result an order was sent out that on admission to the hospital all gassed cases must be cultured for diphtheria.

Three cases came to necropsy in which laryngeal diphtheria was found to be the cause of death but which were not diagnosed clinically. Two more patients died of laryngeal diphtheria in which the diagnosis was made only 24 hours before death. Such cases obviously are a great menace in congested wards. Another case of delayed diagnosis was that of an enlisted man who came to the laboratory for examination accompanied by an attendant from one of the medical huts. He had been sick for 3 days, sitting around the stove in his barracks. There was marked respiratory stridor, some aphonia, and considerable swelling of the neck which had been painted with iodin. On inspection he showed a perfectly characteristic membrane over both tonsils extending up onto the soft palate. Direct smears from the exudate showed the diphtheria bacillus. Such experiences of delayed diagnoses help explain the spread of diphtheria in spite of all other precautions.

\section{BACTERIOLOGY}

During our study of diphtheria at this Center, beside the typical polar-staining bacillus of Klebs and Loeffler, we found a small diphtheroid in large numbers from cultures of the nose and throat. Our attention was called to this organism early, when a ward at base hospital 49 was cultured. Two cases showed this bacillus in practically pure culture with no typical polar staining, and the ward was pronounced negative. Three days later a new clinical case appeared in this ward. This led us to report the first culture, in which this diphtheroid appeared as "Diagnosis reserved," and to request a new culture. If the organism persisted, the report "Positive atypical" was made. This arbitrary standard was to be followed until we had time to obtain results as to its virulence and its frequency in clinical cases.

This diphtheroid is a lancet- or wedge-shaped diplobacillus slightly resembling pneumococcus but somewhat longer in proportion to its width. It shows the palisade arrangement characteristic of the diphtheria group, and tinctorially and morphologically corresponds to " $\mathrm{D}_{2}$ " in Wesbrook's classification. It is gram-positive, nonmotile, shows no spores and no capsule. As a routine in the laboratory we used Barron's polychrome toluidin-blue ${ }^{6}$ diluted $1: 5$, which stains it uniformly except for the midfissure. There are neither barred nor polar forms. We have found that this form may go over into the typical staining organism, may be mixed with it, or may remain entirely true to type. For example, a throat culture has not infrequently been brought to the laboratory in the morning, which after some 8 hours' incubation showed only the wedge forms; whereas, on the following morning only typical polar forms were present. Again, at base hospital 26 one of the gassed cases, followed by pneumonia, was negative when examined in a routine culture of the quarantined ward. Two days later the patient showed a few of the atypical forms, and these were practically in pure culture the next day. A culture on the

- Jour. Lab, and Clin. Med., 1918, 3, p. 432. 


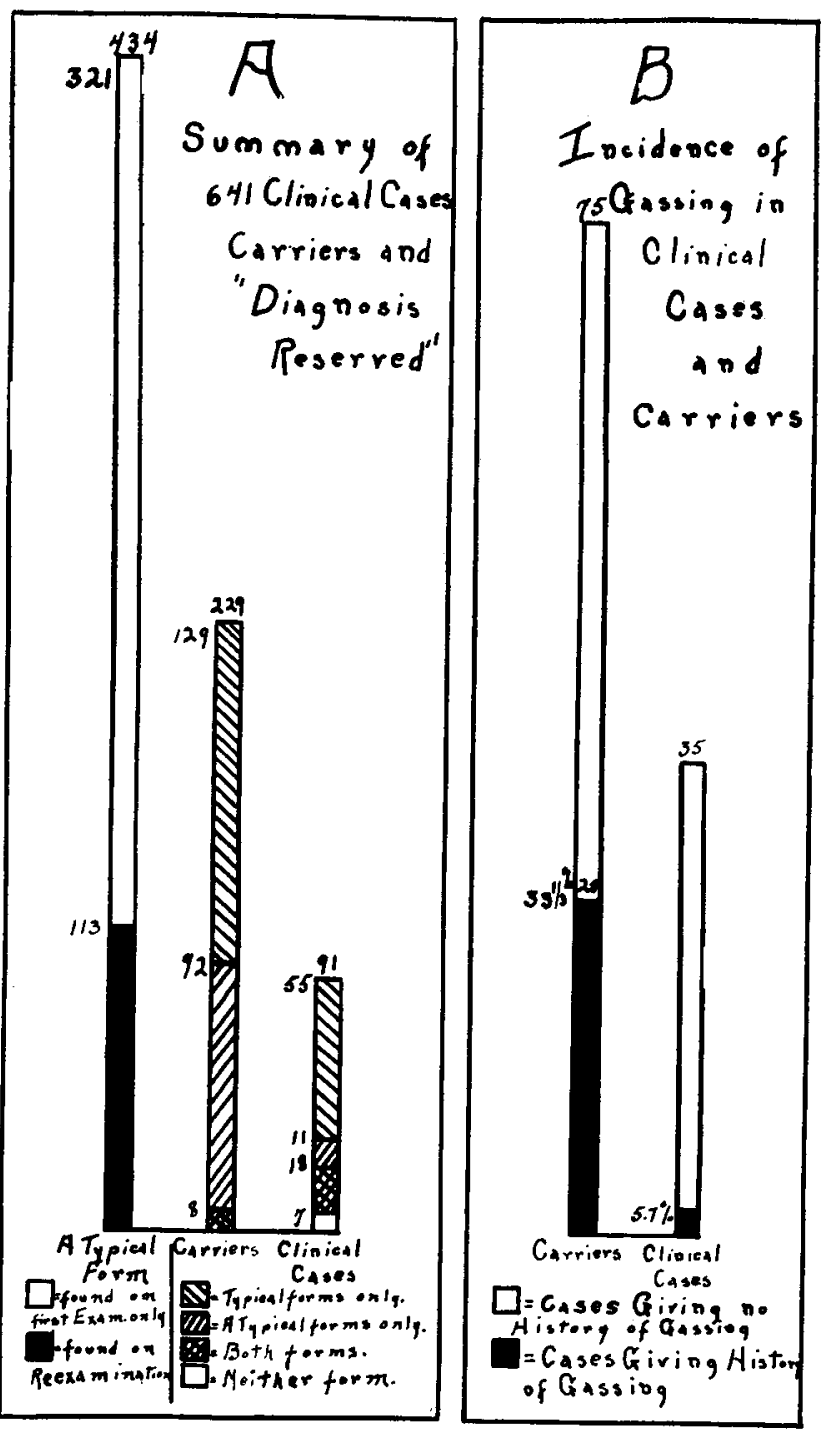

Chart 4.-A: "Diagnosis reserved" was the report given on cultures when the atypical form was first found. A second culture was requested and if the organism persisted the 2nd day the report "Positive atypical" was given. The first column shows that in about three out of every four cases the atypical form did not persist, that is, it was but a transient inhabitant of the throat. Note that the atypical form only was found in $56.1 \%$ of the carriers and $60.4 \%$ of the clinical cases; the atypical form only appeared in $40.1 \%$ of the carriers, and $12 \%$ of the clinical cases; both forms were found at different times in $3.8 \%$ of the carriers and $19.7 \%$ of the clinical cases. The $7.9 \%$ of the clinical cases in which no organism of the diphtheria group was found is accounted for as follows: At first all the individual cultures, among which were most of the clinical cases, were examined at the individual cultures, among which were most of the clinical cases, were examined at the
unit laboratories. If found positive the patient was sent to the contagious hospital, where, owing to the stress of work, no other culture often was taken until the patient was convalescent and ready for discharge. In such cases he would not appear on our records until late and then occasionally as having only negative cultures. B: This chart is made from patients found in the carrier and clinical wards at a given time, and not from selected cases. Unfortunately the percentage of gassed cases of the total number of patients in the hospital was unobtainable. But it is striking that although the carriers and clinical cases cane was the whole But it is st $331 \%$ r from the whole Center at large, $33 \%$ \% of the carriers gave a history of gassing, while only $5 \%$ of the clinical cases gave such a history. 
following day showed typical B. diphtheriae. At necropsy smears from the larynx showed the characteristic organisms, though there was no evidence of any membrane. Some of the throat cultures showing only wedge forms in original culture showed a mixture of wedge, polar, barred and club forms after isolation in pure culture (chart 4).

The cultural characteristics of this form are not unlike those of typical diphtheria. On Loeffler's blood serum, at the end of from 12-16 hours, it shows small, discrete, glistening, buff-gray colonies, slightly raised. Toward the butt of the tube, where the medium is moist, they become confluent. On broth there is moderate clouding, at times flocculation, but no pellicle formation. The relation of this organism to the typical diphtheria bacillus is one of great interest. Table 1 shows the morphology and sugar reactions on 14 cultures isolated early. Six are from clinical cases, of which, in the original cultures, 4 showed the typical organisms, and 2 the wedge form only. When isolated in pure culture all showed the typical form. The other 8 cultures were from carriers, 2 of which showed typical diphtheria, and the 6 others showed a preponderance of the wedge form.

Technic of Isolation.-A small loop of material from the original throat culture was streaked out on one-half of a $1 \%$ dextrose-agar plate, to which one-half of $1 \mathrm{cc}$ of human serum had been added. From this a suspicious colony, usually pinhead size or snaller, discrete, translucent, of a bluish, skimmilk color, was streaked on half of a second plate. From this a colony was streaked on Loeffler's blood serum and after from 16-18 hours' incubation was examined for morphology.

Fermentation Reactions.-Pure cultures were inoculated into tubes of 10 c c of Hiss' serum water to which $1 / 2$ c c of a $20 \%$ sugar solution, sterilized fractionally, had been added. One-tenth of $1 \mathrm{cc}$ of sterile Andrade indicator was added on the 2 nd day. The tubes were read after 2, 4, 6 and 8 days of incubation. On the 8th day smears were examined for contamination.

It has been the experience of one of us in a study of diphtherial infection in wounds that the saccharose fermentation was of considerable importance as regards virulence, that is, that nonsaccharose fermenters are virulent and most saccharose fermenters are avirulent or only slightly virulent. Because of the difficulty in obtaining guinea-pigs and the unsatisfactory results obtained in intracutaneous virulence tests, only five cultures were tested. As chart 4 shows, two of the virulent strains, killing in 24 and 36 hours, respectively, were nonsaccharose fermenters, one being morphologically typical diphtheria, the other showing only wedge forms. The third culture which showed only wedge forms in original throat culture killed in $4 \frac{1}{2}$ days, and fermented saccharose. The other two strains were pure cultures of the atypical organism and had not killed at the end of 2 weeks. All control pigs were negative.

Technic of Virulence Tests.-Forty-eight hour infusion broth cultures were used One-half of $1 \%$ of the body weight of each pig reckoned in $\mathrm{cc}$ was injected subcutaneously. A proportional amount of each culture was injected subcutaneously into a control pig which had been immunized 24 hours previously by an intraperitoneal injection of 100 units of antitoxin. From the pigs that died, cultures were made from the subcutaneous tissues at the site of injection, from the peritoneal fluid, and from the heart blood. The organism injected was recovered only from the one dying after $4 \frac{1}{2}$ days. The adrenals were congested in all three cases. 
TABLE 1

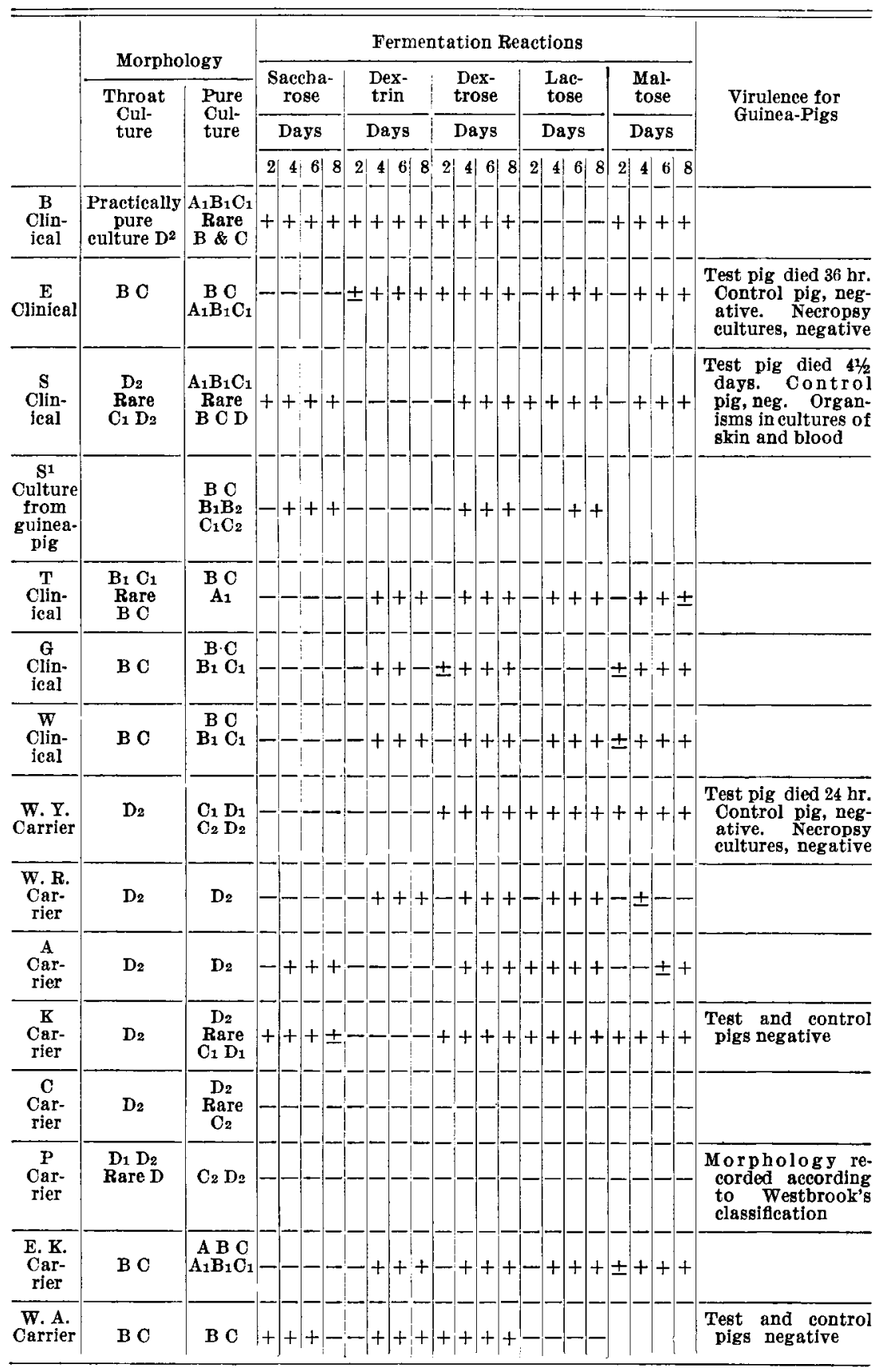

Table 1.-The fermentation reactions are different from those commonly given, in that B. and W. A. ferment both saccharose and dextrin, and ten of the cultures ferment lactose. The two most virulent strains, $E$, and $W$. $Y$. do not ferment saccharose; the less virulent, The most virulent strains, ${ }_{2}$ and morphologically and culturally $\mathbf{B}$. Hoffmanni. Note that the virulent cultures $S$. and $\mathbf{W}$. $Y$. show the wedge form (D-2) predominatingly or in pure cultures in the original throat cultures, while $K$. was avirulent. Cultures $\mathrm{E}$. and $\mathrm{W}$. A., both typically B. diphtheriae, were virulent and avirulent respectively. 
The other data bearing on the virulence of this atypical organism were for the most part clinical. Chart $4 \mathrm{~A}$ gives a summary of our laboratory records up to Dec. 10. Of 434 cases in which the atypical organism was found, only 113 showed it on the following day, that is, in $75 \%$ of the cases it was only a transient inhabitant of the throat. Of the eighteen clinical cases showing both typical and atypical organisms, ten, or $11.1 \%$, of all the clinical cases showed the atypical organism first and later the typical. In eight cases the reverse was true. It cannot be denied that in the ten cases the appearance of the typical form later can be explained by a fresh infection with this organism, since these cases were in the clinical diphtheria ward where cross infection was possible. But in these ten cases the atypical form was able to produce symptoms sufficiently suggestive of diphtheria to warrant their being admitted to the clinical rather than to the carrier ward, and we have had frequent evidence that at times this wedge torm is one of the many forms that may be assumed by the extremely pleomorphic typical diphtheria bacillus.

On Dec. 6, 7 and 8 (chart 1), base hospital 49 showed a sudden increase in the number of atypical forms-one ward having 30 . At the same time base hospital 26 showed a similar increase. On inquiry at the latter hospital it was found that the "team" taking the cultures had become somewhat discouraged at the large amount of work without apparent results, and had decided that it was time for the ward to come out of quarantine. With this end in view they gave up the use of tongue depressors and cultured at random about the tongue and teeth and in the anterior nares. The result was that all three wards cultured on that day showed from 15-25 cases of "Diagnosis reserved." One of these wards, cultured properly, had been entirely negative the day before. No such explanation could be found, however, in the case of base hospital 49. Morphologically this organism found in the mouth and anterior nares could not be distinguished from the wedge form we had been finding in the throat. Also the diphtheroid found on hands and fomites in wards where there had been no diphtheria, had the same morphology.

It is our opin:on that cases showing this atypical organism in properly taken nose and throat cultures must be treated as positive diphtheria cases or carriers until each strain has been proved nonvirulent, since (1) two pure cultures of this organism killed guinea-pigs in 24 and 108 hours, respectively; (2) $11.1 \%$ of the clinical cases showed this form only at first and later showed the typical organism, and (3) variation in length of incubation may show first the atypical and then the typical forms. However, in the majority of cases, this form was only a transient inhabitant of the throat and, we believe, was avirulent.

\section{METHODS OF CONTROL}

From the first, base hospital 56 was chosen as the contagious hospital for the Center and both clinical cases and carriers were sent there. The wards in which these were discovered were quarantined and cultured as directed by the head of the medical service in each hospital. It was early seen that much would be gained both in uniformity of results and in the availability of statistics by having the cultures examined at the central laboratory for the Center rather than by the several unit laboratories. Up to the first of Dec. all the cultures were examined by one man. Since then, owing to the increased 
number, it was found necessary to have two examiners who constantly consulted with each other on doubtful cases.

Toward the latter part of Nov., when from 1-7 new cases developed each day, it was felt that the methods of control at the various hospital units should be more rigorous and more uniform. It was decided to include as clinical cases all those with positive cultures and throat symptoms, though such symptoms might be very slight. Three methods of control were emphasized, any one of which by itself should, theoretically, control spread of infection.

1. Individual Quarantine.-The face ${ }^{7,8}$ mask epitomized individual quarantine. The quarantine of the ward from communication with the outside seemed to be fairly well understood and carried out, but the quarantine of the individuals within the ward from each other was almost completely neglected at that time. For instance, on the discovery of a clinical case the ward was isolated and cultured. Usually one or more positive carriers were discovered and reported in 24 hours. But during this 24 hours the patients who were up would congregate around the stove, owing to inclement weather, playing cards, waiting on the bed patients, and so on. The carriers who were about to be detected had been infecting others by droplet and hand. In other words, at the time when the ward culture was received at the ward it gave no accurate idea as to the number of individuals carrying the diphtheria bacilli in their throats. The same was true of the report on the second ward culture, and so one, ad infinitum. One ward was cultured four times in 7 days. The first culture showed 1 carrier; the second, 2; the third, 2, and the fourth, 4 . After each report the carriers were evacuated to the contagious hospital, and it seemed to be only a question of time and endurance before the entire ward would be transferred.

The $\operatorname{mask}^{9,10}$ was suggested as a remedy. It was, of course, impracticable to mask all of the 16,000 individuals at the Center, though one hospital tried masking its entire personnel. The dissociation of mask from quarantine was found detrimental to the discipline and mental attitude, which is not the least important function of the mask. This might be termed "mask psychology." Cubicles were recommended to supplement the mask, since few can sleep with the

\footnotetext{
7 Capps, J. A.: Jour. Am. Med. Assn., 1918, 70, p. 910.

8 Weaver, G. H.: Ibid., 1918, 70, p. 76.

${ }^{9}$ Doust, B. C., and Lyon, A. H.: Jour. Am. Med. Assn., 1918, 71, p. 1216.

10 Haller, D. A., and Colwell, R. C.: Ibid., 1918, 71, p. 1213.
} 
mask in place. It was urged that the patients be kept by their beds as much as possible, particularly during meals, when, of course, the mask must be removed. The original masks had two layers of gauze with a mesh 14 by 11 threads per $\mathrm{cm}$. It was recommended that two such masks be worn, and that all future masks be made with four layers. Our first point, then, was individual quarantine as represented by mask and cubicle.

2. The Schick Reaction.-Theoretically, if we had examined every individual at the Center by the Schick test and immunized all positives with a prophylactic dose of antitoxin, the spread of diphtheria would have stopped at once. The detection of carriers would have been of no interest, since there would have been no one for them to infect. But this was not considered, because for some time it was questionable whether enough toxin was available for the quarantined wards alone, as had been recommended. A control of inactivated toxin was quite out of the question. The value of the Schick reaction without such a control was the subject of considerable discussion. It was felt that since our object was to stop the spread of the disease at this Center and not to obtain data as to the value of the Schick test, that by reading the reaction at 24,48 and 72 hour intervals we could divide the reactions into three classes: Those frankly positive; those frankly pseudopositive, and those that were questionable. By immunizing cases showing a positive or a questionable reaction, we were erring on the side of safety. On 1,363 Schick tests reported from five of the hospitals, the percentages of positives were as follows: $8 \%$ at threc hospitals, $6 \%$ at one, and $0.6 \%$ at another. Obviously, there was a flaw in the technic of the test at the last named hospital. There were a few carriers showing positive Schick tests, but shortage of guineapigs prevented our testing their cultures for virulence. Also an occasional patient having a negative Schick test came down with diphtheria a few weeks later. The explanations for this are either faulty technic, inert toxin, or overwhelming infection. Our second method of attack, then, was the Schick reaction plus prophylactic immunization.

3. Nose and Throat Cultures.-One of the earliest suggestions for the control of diphtheria was that all of the 16,000 individuals be cultured. Because of our limited autoclave space our daily output of medium could not be pushed beyond 2,000 tubes. There was also a shortage in personnel, both officers and enlisted men. It would, there- 
fore, have taken 8 days at the very least, to culture the entire Center. Thus we would have had the same difficulties which we experienced in the wards before individual quarantine was instituted, only multiplied 8 times, since it was out of the question that during this entire period each individual at the Center would refrain from all except "masked" communication with all the others. At the end of 8 days, our summary report on the names and number of carriers at the Center would have no value, since during the 24 -hour interval between culture and reports, each "positive" would have infected an unknown number of other individuals. So this plan was fortunately abandoned. Definite rules as to the number of times a ward was to be cultured following each new case were formulated. Emphasis was laid on a short, rigorous quarantine since it was felt that the effectiveness of a quarantine is in inverse ratio to its length. Personal cooperation of the patients is absolutely essential to individual quarantine, and this would be entirely lost unless they understood what is being done, why it is being done, and that the hope of release is reasonably within sight.

These rules were helpful not only in standardizing quarantine, but also in stemming the flood of cultures. There is always the danger that wholesale, promiscuous and nonselective culturing may give a false sense of security. For a period of 10 days - from Nov. 29 to Dec. $9-600$ to 1,300 cultures were examined daily. The positives each day varied from 1.1-3.1\% of the total cultures examined. The last 4 days of this period, when the percentage rose as high as $3.1 \%$ a large number of atypical forms was being found, partly accounted for by the careless mouth and anterior nares cultures previously mentioned. During the first six days the percentage varied only from $1.1-1.8 \%$. If from this be subtracted the $0.3-0.6 \%$ of clinical cases each day, there is left a daily quota of carriers amounting roughly to $1 \%$ of those examined. This is what Rosenau ${ }^{11}$ gives as the normal percentage of diphtheria carriers among the unexposed population. In other words, what we really had here was not a potential epidemic of diphtheria but an actual epidemic of culturing. Our third point in control is selective nose and throat cultures, properly taken.

Finally, the most important measure in the suppression of any epidemic is early diagnosis. This is of special significance in a disease such as diphtheria, in which the spread by contact is unquestionable, and, in addition, early diagnosis spells practically $100 \%$ cures.

11 Preventive Medicine and Hygiene, 1918, p. 1374. 
The influence of delayed diagnosis on spread has already been discussed. To emphasize this, a special order was issued that the throats of all patients and personnel be inspected daily. All suspicious cases were to be isolated and cultured. Observation wards were established where such patients were held pending the report on their cultures. Such wards were strictly cubicled and masked, thus reducing contact infection to a minimum.

\section{CONCLUSIONS}

The two probable modes of introduction of the diphtheria bacillus into this Center were "gassed" carriers and chronic carriers.

Factors in spread were crowding (direct droplet infection) contaminated hands and fomites (indirect droplet infection), and delayed diagnosis, partly explained by the similarity in symptoms between laryngeal diphtheria and membranous laryngitis due to gassing. Of these we consider crowding the most important.

Morphology of the diphtheria group is no criterion of virulence. Occasionally, difficulty was encountered in distinguishing between polar-staining, nondiphtheria bacilli and typical B. diphtheriae on morphology alone. Such confusion was experienced only in cultures from hands and fomites, but never in throat cultures.

The four methods of control employed were:

1. Early diagnosis through frequent throat examinations.

2. Individual quarantine exemplified by the mask and cubicle.

3. The Schick test followed by immunization of susceptible individuals with antitoxin.

4. Selective culturing of nose and throat carefully performed. 